The above facts should become known to intending players, so that they may not be misled into thinking that they, will make their fortunes by following the advice given in M. Henry's book. That book adds nothing to our knowledge of the probabilities connected with roulette at Monte Carlo.

\section{THE THEORY OF LIGHT.}

The Theory of Light: A Treatise on Physical Optics. By Richard C. Maclaurin. In three parts. Part i. Pp. viii +326 . (Cambridge: University Press, Igo8.) Price 9s. net.

THIS is the first instalment of a work on optics 1 arranged on a somewhat novel plan. The volume treats mainly of the propagation of light in homogeneous media, isotropic or crystalline, and of the laws of reflection and refraction at plane boundaries. It is to be followed by a second dealing with the subjects of diffraction, dispersion, aberration, \&c.; whilst a concluding volume is to be devoted to the history of optical theory. The method followed is deductive; a medium of the McCullagh type is postulated, and the laws of wave-motion are obtained by an application of the principle of Action. This is practically, of course, the electric theory of light in the form adopted by Larmor. The subsequent developments are naturally almost entirely mathematical, experimental methods being rarely referred to. For this reason the work cannot claim to be, indeed does not profess to be, a complete handbook of the subject; but this is hardly to be regretted, since the English student already has within his reach two masterly expositions from the physical standpoint in Lord Rayleigh's Encyc. Brit. article, and in Prof. Schuster's "Optics." A more serious matter is that some recent speculations of importance are ignored. For instance, we read on p. 29 :-

"The answer forced upon us by the experimental evidence is that we must regard the [components of white] light as polarised elliptically ... for an interval of time which is long compared with the period of vibration, but very short compared with the time required to make any impression on the retina or on a photographic plate."

This brings us back to the standpoint of Airy's "Tracts." It is to be hoped that the author will return to this question in his second volume, and that the bearing on it of Rayleigh's and Schuster's work on interference will receive due consideration.

The real value of the book consists in the systematic mathematical discussion of various classes of phenomena from a common point of view. In particular, many readers will be glad to have in an easily accessible form the author's own investigations of the effect of a thin transition layer in the phenomena of ordinary and crystalline reflection and refraction, and metallic reflection. Regard being had to the point of view, the style is clear and attractive, and the reader will appreciate the numerous excellent graphical representations of the somewhat complicated theoretical results.

In a lively introductory chapter the author discusses the methods and aims of science, the object being apparently to anticipate criticisms which might be directed against the special theoretical basis which he has adopted for his exposition. This discussion is pleasant reading enough, but it is to be hoped that future writers on mathematical physics will not always think it necessary to begin in this way. From the student's point of view the procedure has this disadvantage, that he may find the introduction much harder than the book, and perhaps even not intelligible until he has read the book. In the present instance the opening sentence tells us that "the first question in the catechism of every physicist" should be "what is the chief end of science?" The author's own reply to this question is interesting, and has the present writer's sympathy, but one cannot help wondering what degree of uniformity would be found among the answers which would have been given by, say, Archimedes, Galileo, Newton, Pascal, Laplace, Young, Maxwell, Kelvin. Fortunately history shows that the progress of science is not really conditional on the correct resolution of so formidable a question, any more than art has ever stood still for want of a definitive reply to the other secular question, what constitutes the Beautiful?

The remaining volumes will be looked forward to with interest, and the historical section in particular should prove of great value.

H. L.

\section{GEOLOGICAL EPITOMES.}

Die Alpen. By Dr. Fritz Machaček. Pp. iv +146. (Leipzig: Quelle and Mayer, I908.) Price 1.25 marks.

Eiszeit und Urgeschichte des Menschen. By Prof. Hans Pohlig. Pp. viii + 142. (Leipzig: Quelle and Mayer, 1907.) Price I.25 marks.

THESE two books, bound in cloth and convenient 1 for the pocket, are members of Dr. Paul Herre's series entitled "Wissenschaft und Bildung." They are printed in the older German type, presumably to give them a popular and untechnical aspect; and their cheapness prevents their half-tone illustrations from being more than suggestive. But the text is by no means of the "nature-study " order, or merely intended to lead a young reader on to better things; it is rather a summary of the results of a wide range of specialised research.

Dr. Machaček in his volume compares the views of various authors on the structure of the eastern and the western Alps, and discusses the origin of the present surface-relief. He accepts the theory of glacial erosion for the " Zungenbecken " of the North Italian lakes as a logical outcome of observations on the deepening of the main glaciated valleys further up among the Alps; and he attributes the rich variety of pictorial features (p. $5^{6}$ ) in the central chain to the denuding activities of the Ice-age. Surely no one can nowadays deny the efficacy of "frost-nibbling" in producing crags and cirques and wild arêtes, when combined with the presence of glaciers, which carry off the débris from the scene of severest action. Nor can the modification in form of the original valleys excavated by streams be ascribed to anything but the

$$
\text { No. } 2016 \text {, VOL. } 78]
$$

